\title{
Reframing understanding as a phase transition
}

When understanding comes to us, it can come suddenly. As we finally see how the different parts of a problem fall into place, it can bring about feelings of satisfaction, that we did not discern the seemingly obvious solution beforehand. In their research, Dipl.-Phil. Elena Popova and Professor Valentin Popov at Technische Universität Berlin argue that these drastic shifts in knowledge are a key aspect of the learning process. Furthermore, they terms as phase transitions, as studied in physics.

The human mind has developed many ways to learn new information. Through memory and practice, our minds can gradually become knowledge eventually comes to seem like second nature to us Yet the seaming process does not always happen like this: whether at work, school, or in our everyday lives, we will all at several points have been faced with concepts we are initially unable to wrap our heads around. No matter how much we thought about them, they just did not seem to make sense. After this period of frustration, however, the concepts clicked in our minds all at once, and we finally saw the connections we had missed before. As Professor Valentin Popov, of the Technische Universität Berlin, explains, the reasons underlying this important aspect of the learning process are still poorly phenomenon of learning, but it has always remained a mystery", he says. "A similar phe The most striking property of understanding and discovery is that they may occur suddenly." When this happens, it suddenly becomes obvious how the numerous elements of the problem fit together - often so simply that we the struggle to comprehend how we couldn't see the solution beforehand. As German playwright and scientist Johann Wolfgang von Goethe put it, "the hardest thing to see is what lies in front of your eyes."

SEEING THE FULL PICTURE At first glance, the 1916 painting 'Garden Restaurant on the Havel' appears to be an ordinary scene depicting people . Even after looking at the painting in deta for some time, most observers do not detail: none of the chairs the people are sitting on have any legs 1 To this day, art historians continue to debate whether this detail was an oversight by the artist Max Liebermann, or a well-crafted joke played upon the observer. Regardless of Liebermann's intentions, the painting is a clear example of Goethe's idea in action. Even when our minds have taken in every visual aspect of the painting, we do not seem to process all of the information that was right in front of us. When we finally notice the absence of the chair legs, our experience of the painting is transformed, and we struggle to imagine how we could

"There is a whole course of problems of this kind", Professor Popov explains. "This

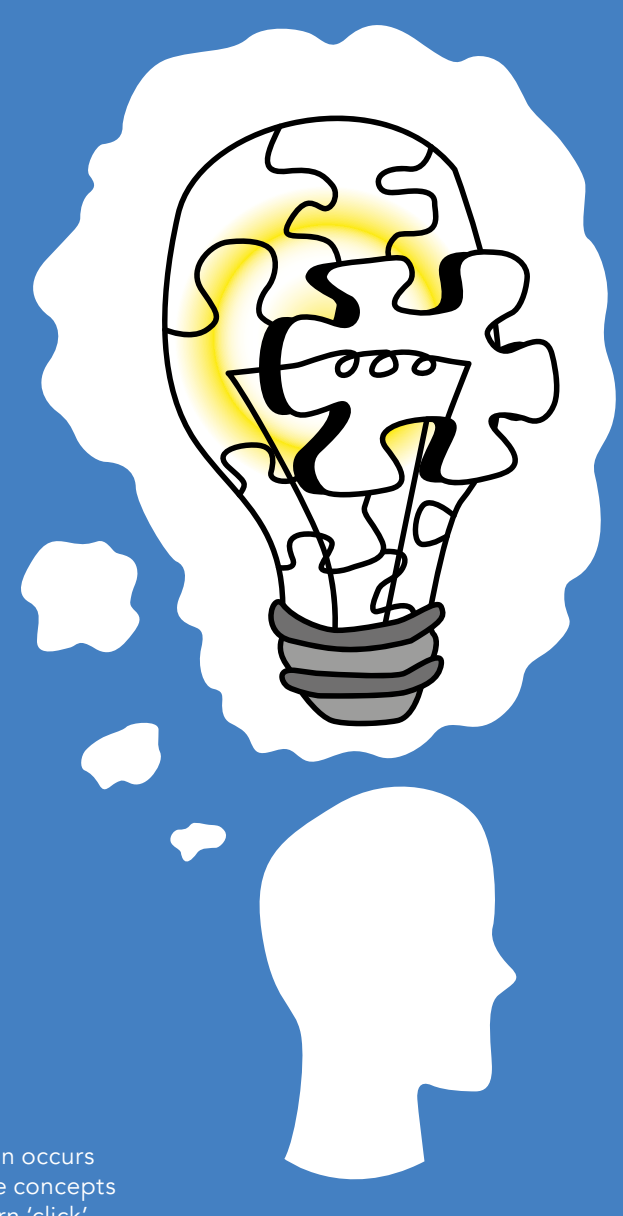

illustrates a very important idea - that understanding is not just a quantitative change in the amount of knowledge, but a special, qualitatively different

bonds or forming new ones. In the specific case of liquid water freezing into ice, molecular arrangements which were suddenly become static and orderly as psychological As Johann Wolfgang von Goethe put it, materials can abruptly phenomenon, this unusual aspect of "the hardest thing to see is what lies in certain 'critical our minds can only front of your eyes".
be explained by the

soon as the water is chilled below $0^{\circ} \mathrm{C}$ Presor Popov and Dipl.-Phil. Popova similar to how numers parts of a

concept can come together in our minds at the abrupt onset of understanding

"The sudden character of understanding shows that this is a critical phenomenon, bringing order to a large number of elements", Professor Popov says. "It transition, establishing a 'long range order' in the corresponding cognitive space." Although the precise neurological processes diving this behaviour are far Po clear, Professor Popov and Dipl.-Ph see the kreyse that we can still clearly taking place in our minds. As such, we can use existing theories of phase transitions, and the establishment of longunderstanding actually works.

\section{ANALOGIES IN}

Materials shifting between solids, only example of phase transitions. A more complex example can be found in superconductivity: an effect which escribes how electrical resistance cerplex

neurological processes which play out Popov and Dipl.-Phil Popova, it can be visualised for more simply by drawing analogies between the sudden onset of understanding and the phase transitions studied in physics.

\section{DRAWING LINKS WITH} PHASE TRANSITIONS

In nature, phase transitions can be readily observed all around us. For example, water can freely transition between being a solid, a liquid, and a gas as it cycles through Earth's atmosphere and experiences widely varying weather conditions. These transitions don't oco gradually: once either temperature or pressure crosses a critical boundary, wa phase; edlately transform to another temperature'. This






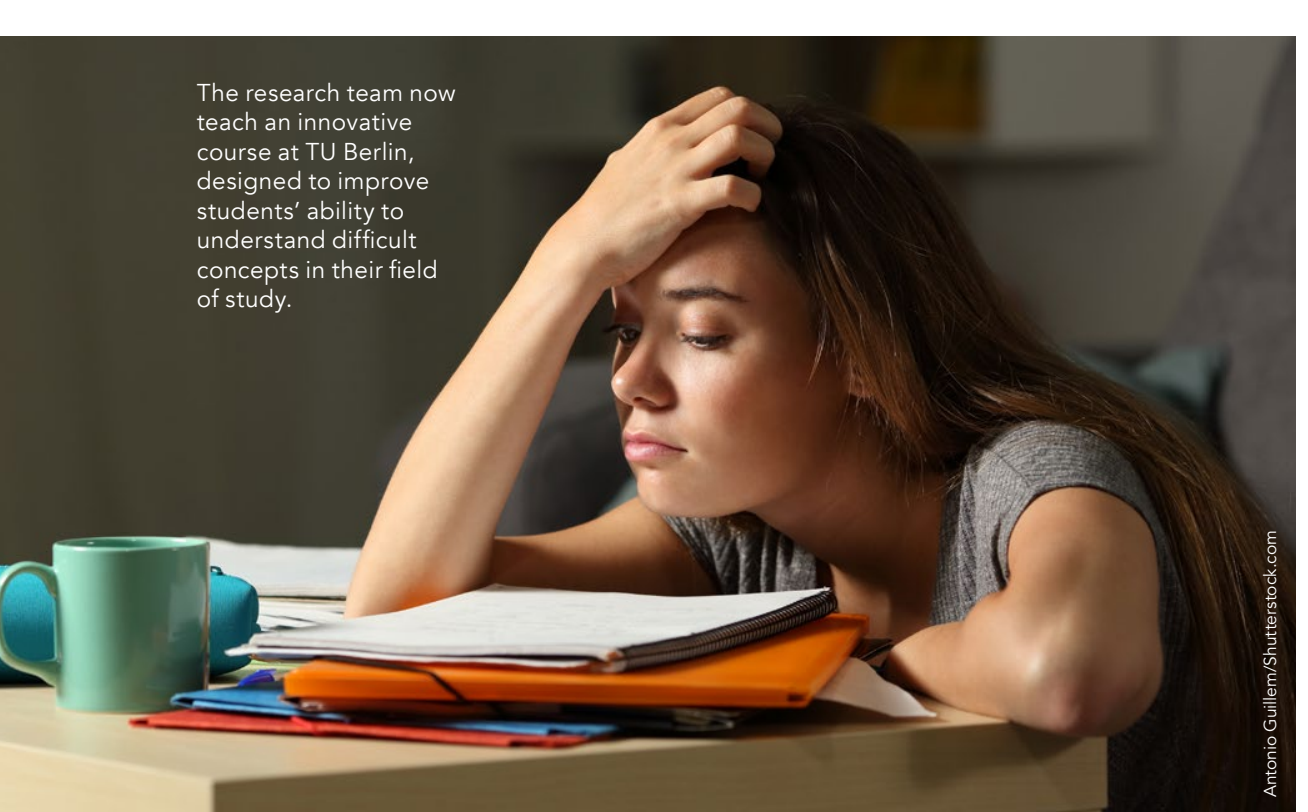

close to absolute zero - the temperature at which all materials will is crossed, the quantum properties of superconducting suddenly transition to a new phase, enabling electrical current to flow even when no power is applied to the materia.

When it was first discovered by Dutch physicist Heike Kamerlingh Onnes in 1911, the very idea of currents
flowing through wires without any flowing through wires without any encouragement appeared to be completely counterintuitive. For decades afterwards, physicists could only observe the effect, without understanding what was really going on. Yet in the late 1950 , new approaches to the analysis of superconducting materials revealed that through measurable, microscopic effects.

"The physical essence appeared to be a phase

with a basic mechanism very similar to other phase transitions, such as the solidification of a liquid", Professor Popov describes. "This shows that an absolutely new quality can also emerg

Until now, the concept of understanding has been largely ignored as an interesting phenomenon in itself.

if the deeply complex mechanisms which drive phase transitions cannot be described in full, their effects can stil be clearly visible - just like the

ACCEPTING UNDERSTANDING AS A UNIOUE EFFECT understanding has been largely

ignored as an interesting phenomeno

students at TU Berlin, designed to mprove their ability to understand the many difficult concepts in their field of study. If the researcher's ideas become more widely accepted in the research vital function: to enhance our serve a see what is really in front of our eyes.

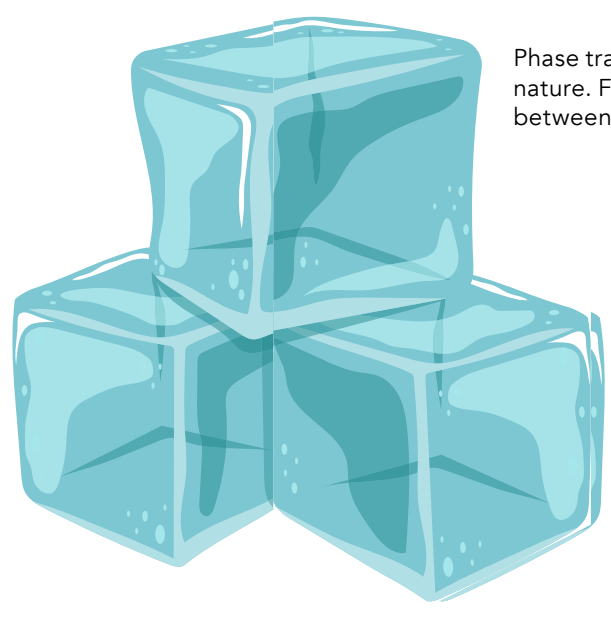

Phase transitions can be readily observed in
nature. Fo hature. For example, water can freely transition
between being a solid, a liquid, and a gas.
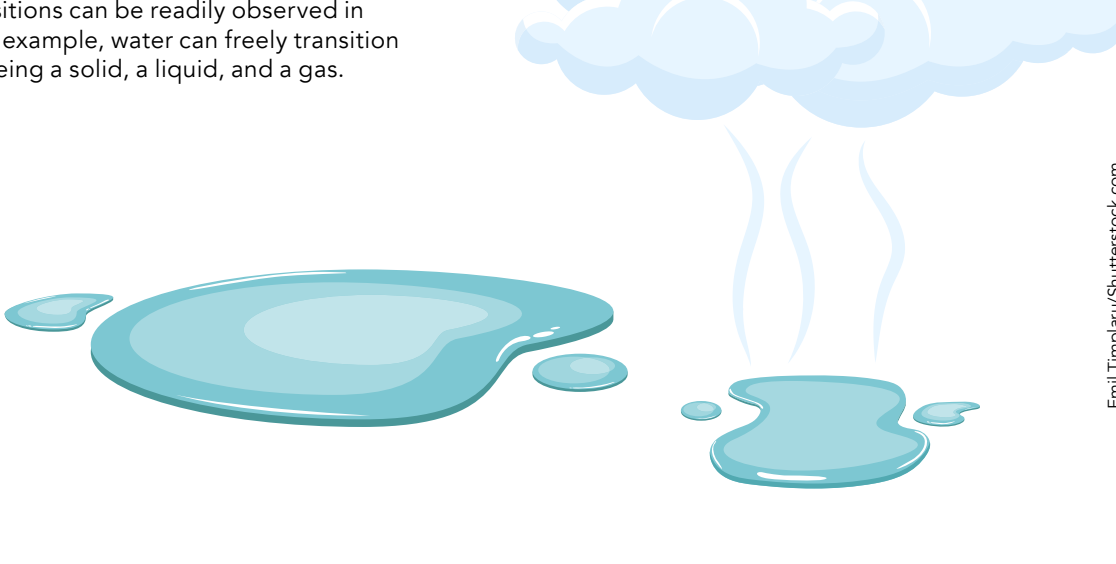

\section{Behind the Research}

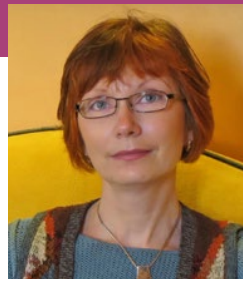

Dipl.-Phil. Elena Popova

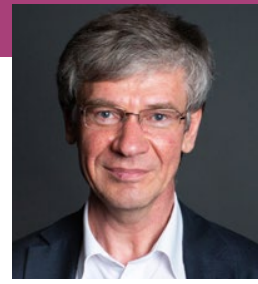

Prof Valentin L. Popov

E: elena.popova@tu-berlin.de E: v.popov@tu-berlin.de T: $+49(30) 31421480$ W: https://www.reibungsphysik.tu-berlin.de/menue/about_us/management/parameter/en/

Research Objectives

Dipl.-Phil. Elena Popova and Professor Valentin Popov are reframing our conception of understanding by describing it in terms of a phase transition.

\section{Detail}

Address

Technische Universität Berlin

Institute of Mechanics

Sekr. C8-4

Str. des 17. Juni 123
10623 Berlin, Germany

Elena Popova, Dipl.-Phil. studied Slavistics at the Tomsk State University, and German literature and Philosophy the University of Paderbonn. She carries out research in the field of history of tribology.

Professor Dr Valentin L. Popov studied physics nd obtained his doctorate from the Moscow State System Dyn mics and Friction Physics at the Tectnische Universiät Berlin since 2002. He is the autor of the book "Contact Mechanics and Friction" having 10

editions in German, English, Russian, Chinese, Spain and apanese and author of a series of papers on the history of tribology.

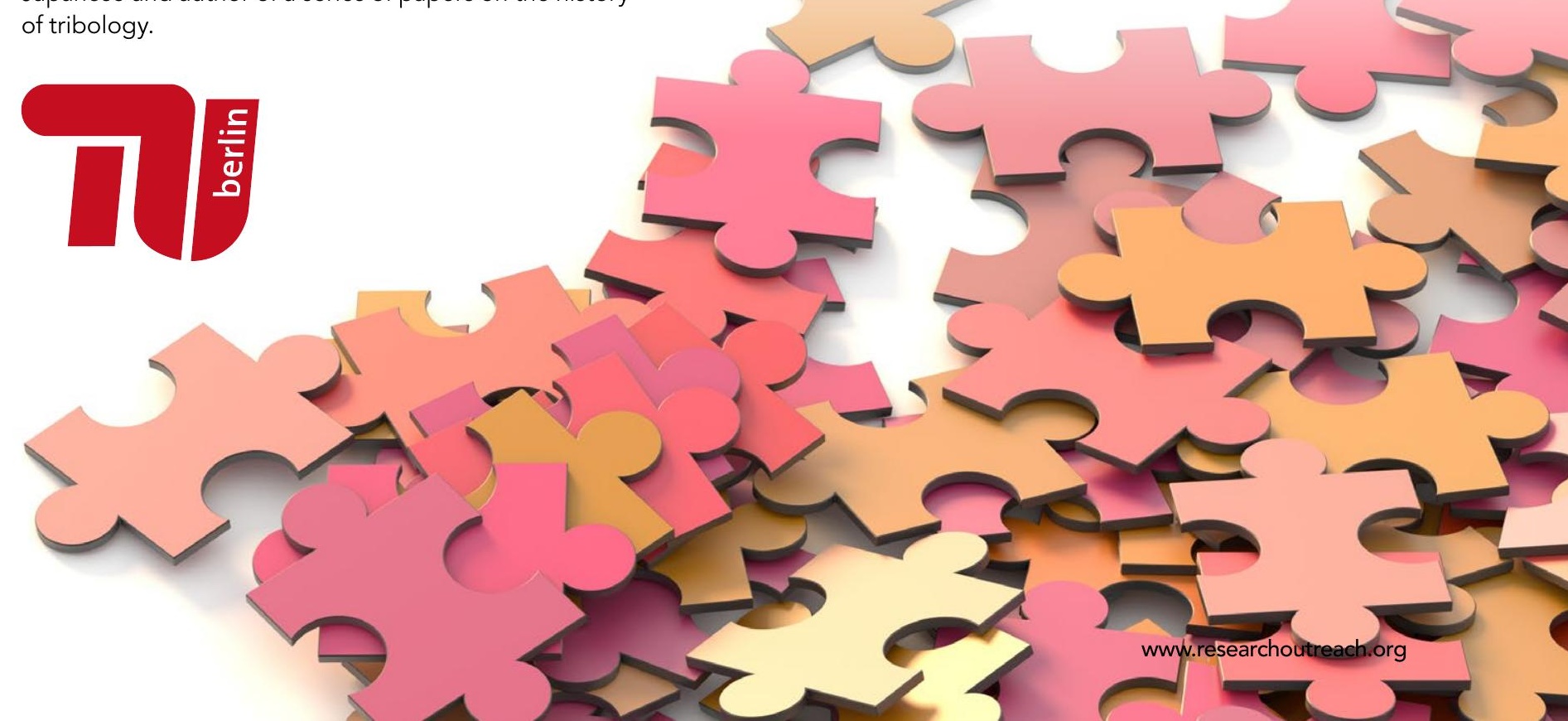

References

Popova, E., Popov, V.L., Filippov, A.E. (2021). Seeing What Lies in Front of Your Eyes: Understanding and Insight in Tribology of Inosganic and Organic Systems, pp. 549-560.

Popova, E., Popov, V.L. (2018). Note on the history of contact mechanics and friction: interplay of electrostatics, theory of gravitation and elasticity from Coulomb to Johnson-Kendall-Roberts theory of adhesion. Physical Mesomechanics, 21(1), pp.1-5.

\section{Personal Response}

Could your insights into understanding allow us to improve the ways we learn new information?

II Prof Valentin Popov: At the Technische Universität Berlin loffer a course that is specifically designed to train the easy to solve". There is nothing to "calculate" there! Once "Ah!" - and that is the solution! In this way, the students get a feeling of what constitutes true understanding. They also gean that preceding hard work is needed and that it is a

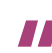
course is that they . "veyp difficul feature of the tasks in this

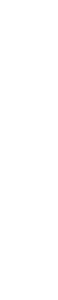

